\title{
Evaluación de competencias en las prácticas de la carrera de Economía de la FAREM Estelí
}

\author{
Evaluation of the competences in the Economy bachelor practice \\ period at FAREM Estelí
}

Reynaldo Gómez García ${ }^{1}$

\section{RESUMEN}

Este documento expone la experiencia de la evaluación por competencias de la carrera de economía en el curso de prácticas de familiarización. El enfoque de competencias se orienta a la enseñanza, aprendizaje y evaluación, centrado en el estudiante, particularmente en los resultados de aprendizaje. En este documento se consideran aspectos conceptuales de prácticas profesionales (y sus etapas), competencias (y términos cercanos), tipos de competencias y la evaluación por competencias. Luego se comparte la experiencia de las prácticas en la carrera de economía y los resultados de una encuesta aplicada a los estudiantes. Desde la perspectiva de los docentes se señalan algunas directrices para una formación basada en competencias, que permita evaluar las competencias de los estudiantes.

Palabras claves: prácticas profesionales; enfoque de competencias; evaluación de competencias.

\begin{abstract}
This paper states the experience of the evaluation by competences of the Economy bachelor practice period. The approach of the competences is oriented to the evaluation, learning and teaching, centered on the students, particularly on the learning results. In this study some conceptual aspects of the professional practice period are considered (and its stages), competences (and related terms), type of competences and the evaluation by competences. Then, the experience of the practice period in the Economy bachelor is shared. Also the results of the survey applied to the students are presented. From the perspective of the professors, some guidelines for competency-based internship are indicated, which allows the evaluation of the student's competences.
\end{abstract}

Keywords: professional practice; competences approach; competences evaluation.

DOI: https://doi.org/10.5377/multiensayos.v3i6.9689

Recibido: 01 de diciembre de 2017

Aceptado: 17 de diciembre de 2017

1 Profesor Titular del Departamento de Ciencias Económicas y Administrativas, UNAN Managua / FAREM Estelí. Correo electrónico: rgomez_garcia@yahoo.com 


\section{INTRODUCCIÓN}

Según Tobón (2008) la formación basada en competencias es un enfoque de gran actualidad e importancia en el contexto educativo y empresarial, ya que aporta bases conceptuales y metodológicas para el desarrollo y afianzamiento de la creatividad, la innovación, la idoneidad, y el espíritu de excelencia dentro del marco de la formación del talento humano que requiere la sociedad.

La evaluación de competencias es un análisis de la capacidad de la persona formada para dar respuestas globales y diferentes según las diversas situaciones profesionales que enfrente (Gairín, Armengol, Gisbert, García-SanPedro, Rodríguez y Cela, 2009, p.10). Para la Universidad Nacional Autónoma de Nicaragua, Managua (UNAN-Managua, 2011, p.73) una de las principales tareas del quehacer educativo en la educación superior es la evaluación continua de los proyectos y programas educativos.

La formación y evaluación de competencias en la carrera de economía es útil para el aprendizaje significativo y la formación integral de los estudiantes. Es una integración de la teoría con la práctica, que trasciende los niveles educativos, buscando el desarrollo de un espíritu emprendedor para el crecimiento personal. En ese sentido, sobrepasa los currículos donde las asignaturas se agrupan por áreas de formación, fragmentando el conocimiento.

En el presente ensayo, lo que se pretende es compartir la experiencia de la evaluación de las competencias desarrolladas por estudiantes de economía de la Facultad Regional Multidisciplinaria Estelí (FAREM Estelí) de la UNAN Mangua, en la asignatura de prácticas de familiarización. Sin embargo, antes de entrar en materia primero se abordarán elementos conceptuales de prácticas profesionales, formación basada en competencias, evaluación de competencias, y posteriormente se compartirán algunos resultados del proceso evaluación de competencias de los estudiantes de economía.

\section{DESARROLLO}

La UNAN Managua (2011) conceptualiza el desarrollo de las prácticas profesionales como "una práctica académica estudiantil, que contribuye a consolidar los conocimientos, habilidades y destrezas adquiridas por los y las estudiantes, así como el desarrollo de actitudes y valores" (p.68). Son parte sustancial del Plan de Estudios y tienen el objetivo de vincular interdisciplinariamente al estudiante en su futuro campo laboral.

Las Prácticas Profesionales se convierten en una actividad obligatoria, dirigida a fomentar el contacto con la realidad, facilitando la incursión del estudiante en el mundo laboral. Esta actividad académica favorece el proceso de formación integral del estudiante, a través del contraste entre el conocimiento teórico (inter y multidisciplinar) y la actividad práctica que la realidad proporción. 
Esto permite la vinculación de la Universidad con el entorno social y productivo (UNAN Managua, 2011).

Todas las carreras de la UNAN-Managua contemplan en sus planes de estudios las prácticas profesionales, que se componen de tres etapas:

- Prácticas de familiarización: es aquella donde se prepara al estudiante y se le va familiarizando con el ambiente laboral y profesional en el que se desenvolverá una vez terminado el proceso de formación profesional. Esta etapa le permitirá conocer los pormenores de su profesión, así como los obstáculos a los que se enfrentará en la misma.

- Prácticas de especialización: esta fase permitirá al estudiante definir el área de especialización, que más le interesa, ya que según el perfil profesional tienen a posibilidad de desempeñarse en varias áreas. Con estas prácticas tendrá la oportunidad de relacionar la teoría con la práctica, lo que a su vez, garantiza, la aplicación del conocimiento científico adquirido en los diferentes campos de su profesión. Esta fase brinda la posibilidad de llevar a cabo proyectos de atención a necesidades especificar en una área de especialización profesional.

- Prácticas de profesionalización: es aquella donde el estudiante ha de aplicar todos los conocimientos, habilidades, destrezas, actitudes y valores desarrollados durante su formación.

El concepto de competencias es utilizado desde hace tiempo en el campo de formación profesional, para luego abrirse camino en todo el sistema educativo y en los procesos de formación continua, donde la formación por competencias se ha convertido en un modelo integrador y orientador de las diferentes políticas de recursos humanos (Gairín, 2010).

Debido a que las competencias tienen su origen en el sentido de la formación y su vinculación con la actividad laboral, las reformas educativas enfatizan en lo laboral, dejando de un lado la formación de competencias personales y para la convivencia social. En ese sentido, las nuevas orientaciones de los planes de estudio parecen omitir los procesos reflexivos personal y despreciar la cultura general.

Existen diversos conceptos cercanos al término de competencia que no deben prestarse a confusión, tales como (Tobón y Fernández-Salinero citado por García San-Pedro, 2009):

- Cualificaciones personales: capacidad general de desempeñar todo un conjunto d tareas $\mathrm{y}$ actividades relacionadas con un oficio y apoyadas en una certificación acreditada. Las competencias enfatizan su desempeño en procesos personales y profesionales específicos, donde lo central es la adaptación al cambio y flexibilidad en el desempeño de actividades.

- Capacidades: condiciones cognitiva, afectivas y psicomotrices fundamentales para aprender y que denotan la dedicación a una tarea. Son el desarrollo de las aptitudes. Las competencias tiene como componentes las capacidades con el fin de llevar a cabo las actividades.

- Destrezas: mediadores entre las capacidades y las habilidades y cuya adquisición supone el 
dominio tanto de la percepción frente a los estímulos de la reacción eficaz para ejecutar la tarea. Las competencias considera la destreza como base para la actuación, pero difieren de estas porque integran el conocimiento, los procedimientos y las actitudes en la búsqueda de objetivos de corto y largo plazo.

- Habilidades: consiste en cualidades que permiten realizar tareas y actividades con eficacia y eficiencia. Las competencias integran la compresión de la situación, conciencia crítica, espíritu de reto, responsabilidad por las acciones y desempeño basado en indicadores de calidad.

- Actitudes: disposiciones afectivas para la acción que constituyen el motor que impulsa al comportamiento en los seres humanos. Induce igualmente a la toma de decisiones y a desplegar un determinado tipo de comportamiento acorde a con las circunstancias del momento. Las competencias de componen de cuatro saberes (conocer, hacer, estar y ser), de los cuales el saber ser está integrado por valores, estrategias psico-afectivas y actitudes.

La competencia es la "combinación de habilidades, actitudes y conocimientos necesarios para desarrollar una tarea de una manera eficaz" (Gairín y otros, 2009, p.17). Estas competencias son aprendidas y se desarrollan a partir de actividades que permiten integrar habilidades, actitudes y conocimientos aprendidas anteriormente, que se demuestran en la acción.

Según Gairín (2010) las competencias se identifican con características de las personas con una actuación de éxito en su lugar de trabajo. Se desarrollan a partir de experiencias de aprendizaje integrativas en las que conocimientos de diversa naturaleza, habilidades y actitudes interactúan con el fin de dar una respuesta eficiente en la tarea que se ejecuta.

Dependiendo del tipo de titulación, se pueden diferenciar dos amplios grupos de competencias (Gairín y otros, 2009, p.18):

- Competencias específicas, son propias de un ámbito o titulación y están orientadas a la consecución de un perfil especifico del graduado o graduada, Son próximas a ciertos aspectos formativos, áreas de conocimiento o agrupaciones de materias, por eso tienen una proyección longitudinal en la titulación.

- Competencias genéricas (transversales), son comunes a la mayoría de los títulos aunque con una incidencia diferente y contextualizada para cada titulación.

Dentro de este último bloque están las siguientes competencias:

- Competencias personales, como la gestión del tiempo y la responsabilidad del mismo aprendizaje.

- Competencias interpersonales, como comunicarse, trabajar en equipo, liderar o negociar.

- Competencias relacionadas o instrumentales, como la gestión de la información, los idiomas e informática. 
El desarrollo efectivo de las competencias depende de las oportunidades para ponerlas en práctica e implica un programa estructurado y coherente a lo largo de todo un plan de estudio. Por eso, es importante la integración de las competencias al currículo universitario para la mejora en el aprendizaje de los estudiantes mediante el desarrollo de estrategias que promuevan y evalúen las competencias.

La evaluación por competencias se integra a tres niveles (García San-Pedro y Gairín, 2011): nivel institucional, nivel de programas y nivel de cursos. El nivel de curso o materias se dan en el contexto de una disciplina y las competencias se integran a través de una plantilla o mapa. El mapa de competencias es un instrumento a través del cual se puede observar que competencias son desarrolladas, lo momentos en que se desarrollan, la carencia de espacios curriculares, los solapamientos y las fortalezas.

El aprendizaje basado en competencias asegura que los estudiantes adquieran aquellos conocimientos, habilidades y actitudes importantes tanto para sus estudios como para su vida laboral. La aplicación de competencias implica el desarrollo de cuatros componentes:

- Descripción de la competencia, es comunicar a los estudiantes lo que se pretende alcanzar con el proceso de enseñanza-aprendizaje. Es el referente de que saben los graduados y que son capaces de hacer.

- Descripción de la actividad en que se manifestará la competencia, es explicitar que conocimientos y habilidades llevan implícitos y en qué contexto se aplicará, así como su nivel de profundidad o complejidad.

- Instrumentos o medios para evaluar las competencias, la competencia se evalúa en la acción y para adquirirla son necesarios una serie de conocimientos, habilidades y actitudes previas. Por eso, la pirámide de Miller es un instrumento para escoger estrategias de evaluación coherentes con los resultados de aprendizaje descritos.

- Estándares o criterios para juzgar si alguien es o no competente, es establecer criterios valorativos que permitan emitir juicio de valor respecto a los resultados alcanzados. Es aclarar los aspectos o las dimensiones que se quieren evaluar, así como los indicadores o evidencias que identifique los niveles de valoración.

La diversidad de estilos de aprendizaje y la variedad de competencias exige estrategias y procedimiento de evaluación diversos, algunos centrados en los estudiantes (autoevaluación y evaluación entre iguales) y otros centrados en el docente (exámenes, informes, exposiciones, etc.).

La evaluación no es un hecho aislado del proceso formativo, sino que constituyen una parte fundamental de este proceso. En ese sentido, las siguientes estrategias e instrumentos aportan información y evidencias valiosas a la hora de tomar decisiones: 
- Debate y grupo de discusión: es un trabajo en grupo que tiene como objetivo principal que el estudiante analice y busque soluciones o aclare sus ideas en torno a un problema o situación determina, a partir de la exposición e intercambio de opiniones o puntos de vistas. Un instrumento para evaluar dicha actividad es una ficha de evaluación sistemática.

- Seminario: basado en el trabajo en grupo e intercambio de ideas, que persigue la reflexión, la discusión y el análisis en profundidad de un tema decidido previamente.

- Prácticas de aula: actividades hechas por el estudiante durante la hora de clase que tiene como objetivo consolidar los conocimientos teóricos desarrollados en el temario.

- Resolución de casos: se basa en la presentación y la descripción de una situación real o ficticia, donde el estudiante tiene que analizar la situación, identificar los problemas y decidir las acciones que se tendría que aplicar para resolver el caso. Un instrumento para evaluar el grado de colaboración es el cuestionario de autoevaluación.

- Proyecto: actividades sistemáticas y elaboradas que se llevan a cabo con el fin de dar solución a un problema o pregunta. Un posible instrumento puede ser un cuestionario que valore cada uno de los elementos del proyecto.

- Exposición oral: consiste en la presentación organizada de información ante una audiencia. En una exposición es conveniente evaluar aquellos aspectos de la comunicación mediante pautas de observación

- Prueba objetiva: basada en la formulación de un conjunto de preguntas breves que van acompañadas de diversas alternativas de respuestas.

- Exámenes comprensivos: incluye un conjunto de preguntas abiertas, referente a un tema o aspecto concreto, que tienen que ser desarrolladas por el estudiante.

- Articulo o ensayo: escrito mediante el que se desarrollan las ideas y el conocimiento en relación a un tópico sugerido por el docente o que él mismo ha escogido. Esto favorece el desarrollo de la reflexión crítica y de la expresión escrita.

Una vez abordados los elementos teóricos-conceptuales sobre prácticas profesionales y competencias paso a realizar un recorrido por el plan de estudio y programa de asignatura, para luego compartir la experiencia de cómo se evaluaron las competencias desarrolladas por estudiantes de economía en sus prácticas de familiarización durante el II semestre de 2017, de la FAREM Estelí.

Según el plan de estudio de la carrera economía y el programa de la asignatura de prácticas de familiarización se ubican en el tercer año y sexto semestre de dicha carrera. Se trata de un curso teórico e instrumental que está precedido por 12 asignaturas y que tiene como propósito general contribuir a una visión teórico-cuantitativa de la economía y dimensión social, que contribuya al desarrollo de las capacidades conceptuales, procedimentales y actitudinales de los estudiantes para una mejor compresión de los fenómenos históricos y económicos.

Por otro lado, en el descriptor de la asignatura se establece que las prácticas de familiarización permitirán a los estudiantes desarrollar conocimientos generales para el aprendizaje de la economía, 
así como habilidades intelectuales relacionadas con la economía y los sectores económicos. Adicionalmente, los estudiantes adquirirán habilidades de comunicación interpersonal, de organización y gestión personal.

La asignatura de prácticas está organizada en cinco unidades: producción; precios; empleo y salarios; moneda y bancos. En su fundamentación o mapa de la asignatura se establecen una serie de capacidades a desarrollar en las unidades, tales como:

- Determina el objeto de estudio de los sectores económicos;

- Define conceptos económicos;

- Analiza los principales indicadores de la economía;

- Describe la actividad económica;

- Identifica los principales rubros de la economía;

- Interpreta indicadores económicos;

- Redacta informes, ensayos y artículos económicos; y

- Aprecia la actividad económica a través de los sectores económicos.

Las capacidades antes mencionadas se resumen en tres funciones básicas:

- Realizar análisis económico.

- Analizar indicadores de la economía.

- Investigar la actividad económica.

Para cumplir con las funciones y capacidades expuestas se consideran las recomendaciones metodológicas por unidad, que están en correspondencia con el modelo educativo, modelo curricular y modelo pedagógico de adoptado por la UNAN Managua.

Las estrategias de enseñanza-aprendizaje expuestas en el programa de asignatura se sustentan tanto en las modalidades como en sus métodos. Las modalidades propuestas son: clases teóricas; seminario-taller; clase práctica, tutorías, trabajo en grupo y autónomo. Los métodos son: expositivos; estudios de caso; resolución de ejercicios y problemas; aprendizaje orientado a trabajos, aprendizaje cooperativo y compromiso de aprendizaje.

A pesar de que la UNAN Managua aplica un currículo por objetivos (conceptuales, procedimentales y actitudinales), estos se vinculan a los criterios de desempeño (saber conocer, saber hacer y saber ser) para el desarrollo de competencias. Estos objetivos son plasmados en los planes didácticos y se detallan en los planes diarios de clase.

¿Cómo evaluó las competencias en estudiantes de prácticas de familiarización? Aunque, en el programa de asignatura existen recomendaciones metodológicas. Con base a mi experiencia docente y profesional, trabajo de la siguiente manera: 
- Unidad 1: Producción, el contenido se enfoca a la medición de la producción nacional mediante el producto interno bruto (PIB), ya que los estudiantes conocen el concepto y saben calcular el PIB, ahora les enseño a buscar en la página web del Banco Central de Nicaragua (BCN) el PIB según los enfoques de producción y de gasto, para que ellos a través de Excel aprendan a elaborar tablas y gráficos para realizar un análisis de tendencia. La modalidad es de trabajo en grupo y el método aprendizaje orientado a trabajos.

- Unidad 2: Precios, el contenido se vincula al índice de precios al consumidor (IPC), como los estudiantes ya están familiarizado con el concepto IPC, entonces les enseño cómo construir un IPC a partir de una canasta de 20 productos de primera necesidad en su hogar. Ellos aprenden mediante el empleo de Excel a darle seguimiento al comportamiento de la inflación por medio de la cotización mensual de esos 20 productos seleccionados. La modalidad de trabajo es autónomo y los métodos son aprendizaje orientado a trabajos y expositivos, porque al final del semestre deben entregar un informe y defenderlo.

- Unidad 3: empleo y salarios, este contenido es referente al mercado laboral (tasa de empleo y desempleo) y la ley de salario mínimo, debido a que muchos estudiantes no manejan estos conceptos, inicio con una clase teórica-conceptual sobre los términos del mercado laboral. Luego procedo a la asignación de un municipio a cada grupo para que lo caractericen, es parecido a una consultoría técnica, que emplea cálculos en Excel y finaliza con la defensa del informe final. Para tal fin, deben buscar información en las páginas del Banco Central de Nicaragua (BCN) y del Instituto Nacional de Información de Desarrollo (INIDE). La modalidad es de trabajo en grupo, los métodos son aprendizaje orientado a trabajos y expositivos.

- Unidad 4: Moneda, es un contenido de dinero y tasas de interés, aunque los estudiantes ya vieron algunos de estos términos en las clases de macroeconomía, lo que hago es reforzar aquellos conocimientos donde hay lagunas y profundizo en análisis de la tasas de interés (pasiva, activa y margen bancario), para que los estudiantes aprendan hacer tablas y gráficos con su respectivo análisis de tendencia. La modalidad es de trabajo en grupo y el método aprendizaje orientado a trabajos

- Unidad 5: Bancos, los contenidos están enfocados al sistema financiero, ya que los estudiantes tienen conocimiento general de cómo funciona el sistema financiero en Nicaragua, por eso aprovecho para asignarles una institución financiera (bancos, financieras, cooperativas de ahorro y crédito, bolsa de valores, compañías de seguro, fondos de pensiones, almacenes de depósitos, leasing y factoring) a cada grupo para que profundicen en su estudio (funcionamiento e importancia dentro del sistema). Estas investigaciones, permiten el estudio de la actividad económica. La modalidad es de trabajo en grupo, los métodos son aprendizaje orientado a trabajos y expositivos. 
La evaluación de la asignatura de prácticas de familiarización es sistemática, porque durante todo el curso se va evaluando el avance que presenta cada estudiante, aunque empezamos con prácticas dentro y fuera del aula, hay espacio para el debate y la discusión, así como en tres de las cinco unidades existe exposición oral, es decir, que se emplea una variedad de estrategias de evaluación de competencias de los estudiantes.

Al final del curso, se aplicó una encuesta a 10 de los 29 estudiantes que cursaban la asignatura de prácticas de familiarización, para determinar su percepción con respecto al nivel alcanzado en 12 competencias previamente establecidas en tres categorías: cognitivas, técnicas y socioemocionales. Los resultados indican que los estudiantes valoran con alto desarrollo de competencias el uso de programas básicos de informática, la búsqueda de información, el uso de la TIC y la iniciativa/ motivación, pero con bajo o nulo desarrollo la comunicación escrita y la expresión oral.

\section{CONCLUSIONES}

La evaluación de competencias es un análisis de la capacidad de la persona formada para dar respuestas globales y diferentes en dependencia de la situación profesional a que se enfrente. Sin embargo, la dificultad de estructurar acciones de evaluaciones en contexto laboral se pueden contemplar como una dificultad o una limitación al evaluar competencias en la universidad. Por eso, es necesario el trabajo en equipo de los docentes para una misma competencias se pueda desarrollar desde diferentes materias o módulos formativos.

En la asignatura de prácticas de familiarización, los estudiantes logran desarrollar ciertas competencias y reforzar otras a través de un aprendizaje fundamentado en el aprender haciendo. En un aprendizaje para la vida que les servirá en diferentes contextos, empezando con el aprendizaje autónomo y en grupo, para llegar al aprendizaje cooperativo. A pesar de estos esfuerzos, aún existen algunas barreras a superar, como la poca o nula articulación de las asignaturas y del trabajo en equipo de los docentes, lo cual limita el aprendizaje integral del estudiante.

Es imprescindible la capacitación e inter-capacitación de los docentes para compartir sus experiencias (saberes acumulados) y el desarrollo de competencias en el profesorado. Estos elementos deben conducir a un equilibrio razonable, que no incline la balanza hacia docentes formados o fogueados solo en la academia (saber conocer) o hacia docentes formados en el ámbito laboral (saber hacer) con desconocimiento del currículo, lo cual contribuirá una verdadera formación basada en competencias. Además, la UNAN Managua va encaminada hacia ese currículo por competencias, donde de una u otra forma estaremos involucrados.

\section{BIBLIOGRAFÍA}

Gairín, J. (2010). La formación de profesorados basado en competencias. Barcelona: Universidad de Barcelona. 
Gairín, J., Armengol, C., Gisbert, M., García-SanPedro, M. J., Rodríguez, D., \& Cela, J. M. (2009). Guía para la evaluación de competencias en el área de ciencias sociales. Barcelona: Agència per a la Qualitat del Sistema Universitari de Catalunya.

García-SanPedro, M. J. (2009). El concepto de competencias y su adopción en el sistema universitario. Revista alternativa. Cuadernos de trabajo social, 11-28.

García-SanPedro, M. J., \& Gairín Sallán, J. (2011). Los mapas de competencias: una herramienta para mejorar la calidad de la formación universitaria. Revista Iberoamericana sobre Calidad, Eficacia y Cambio en Educación, 84-102.

Tobón, S. (2008). Formación basada en competencias. Bogotá: Ecoe Ediciones.

UNAN-Managua. (Septiembre 2011). Modelo Educativo, Normativa y Metodología para la Planificación Curricular 2011. Managua: Consejo Universitario de la UNAN Mangua. 